\title{
ItsPhone: An Integrated Platform for Participatory ITS Data Collection and Opportunistic Transfer
}

\author{
O. Briante*, C. Campolo*, A. Iera*, A. Molinaro*, S.Y. Paratore*, G. Ruggeri*, M.J. Booysen ${ }^{\dagger}$ \\ *University Mediterranea of Reggio Calabria, Italy, E-mail: giuseppe.ruggeri@unirc.it \\ ${ }^{\dagger}$ Department of Electrical and Electronic Engineering, Stellenbosch University, South Africa, E-mail: mjbooysen@sun.ac.za
}

\begin{abstract}
The recent advances in sensing and communication technologies pave the way for interfacing cars with smartphones to offer value-added services to users on the road. Kinematics, automotive diagnostics, passengers load, and pollution data can be provided by vehicles, augmented with external sensors, to make the road transport greener and smarter.

With the purpose of supporting and improving data collection and distribution, by boosting user participation, in this work a smartphone-based platform is demonstrated that exploits cheap dedicated hardware to interact with sensors on board and in the vehicle's surroundings and opportunistically leverages available wireless connectivity to remotely transmit collected data.
\end{abstract}

\section{INTRODUCTION}

In the recent years there has been a surge of interest from academia, governmental agencies, and road authorities in addressing the challenging issues of traffic congestion and increased pollution. These problems can be largely alleviated by exploiting Intelligent Transportation Systems (ITS) solutions, which require a strong synergy between sensing and communication technologies to support the effective and efficient data collection and distribution between vehicles and the infrastructure. Today there are many opportunities to allow citizens on wheels to take part in the collection and remote upload of ITS data, by leveraging the new technological features of modern vehicles and wireless devices and the interesting opportunities of crowdsourcing.

To this purpose, we propose and demonstrate ItsPhone, Integrated plaTform to Support Participatory ITS data cOllection and opportuNistic transfEr, an easy-to-use framework devoted to the collection and remote delivery of a variety of information about the vehicle status and its surroundings, Fig. 1. ItsPhone emerges as a short term solution enabling ITS applications deployment. In fact, by leveraging popular smartphones, equipped with a positioning system and multiple wireless network interfaces, additional cheap hardware and opensource software, the proposed platform can be adopted on a large-scale by citizens and commuters willing to contribute to data collection while benefiting from the value-added onthe-road services (e.g., access to real-time traffic information, alternative route suggestions, connection to dealerships for service tips, etc.).

By using the ItsPhone features, kinematics and diagnostics data provided by the in-vehicle Controller Area Network

This work has been carried out under the framework of the international research project "PALMARES: an Internet of Smart Objects", funded by the Italian government within the Cooperlink initiative.

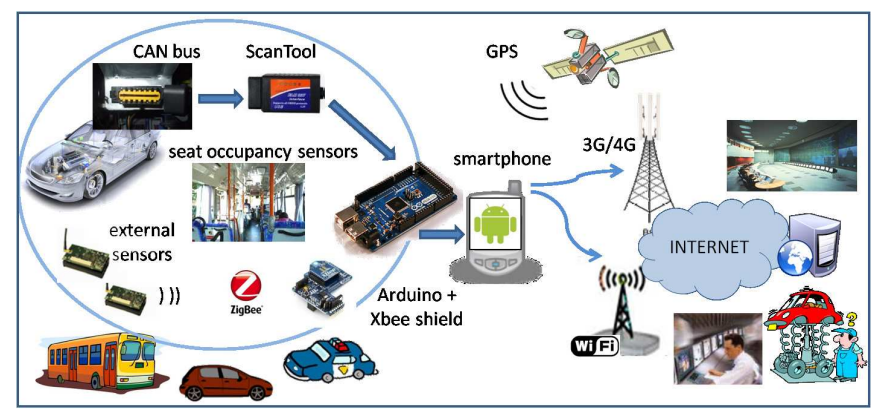

Fig. 1. The reference scenario for the ItsPhone platform.

(CAN) bus can be transmitted to traffic management servers. Through additional sensors in the envisioned platform, vehicles may collect data relevant to passenger load status and environmental conditions and make them remotely available for monitoring and processing purposes.

Recently a great interest is arising in developing automotive applications based on smartphones. In [1], an Androidbased smartphone monitors CAN bus data from an On-board Diagnostics (OBD) interface ${ }^{1}$ and if an accident occurs, it is promptly notified. In [2], a smartphone application is designed that uses sensors to enable detection of the driving pattern, and suggests new behaviors to reduce the fuel consumption. In [3] smartphones and human inputs are jointly exploited to collect ITS data.

Despite such efforts in application and interface development, contributions are still missing related to the design of a ready-to-use platform that $(i)$ properly collects ITS data from heterogeneous sensors and (ii) opportunistically transfers them, while meeting their delivery constraints and offloading the cellular network. In fact, despite the low data footprint per vehicle (up a few hundreds bytes/per second), the amount of generated data from different contributing users is expected to heavily load the cellular network together with the upcoming machine-to-machine data traffic. In order to manage this issue and offload the cellular network, the conceived ItsPhone platform is augmented with opportunistic data delivery functions, leveraging intermittent Wi-Fi connectivity and the delaytolerant nature of some collected data.

\footnotetext{
${ }^{1} \mathrm{OBD}$ is the standard that specifies the type of diagnostic connectors and OBD-II is made mandatory for all cars sold in the US since 1996.
} 


\section{System DESIGN AND IMPLEMENTATION}

The two main ItsPhone building blocks are: (1) the Data collection and pre-processing module, realized through a dedicated USB-enabled microcontroller board, Arduino [4], receiving inputs from a variety of in-vehicle and external sensors, and responsible for merging and temporarily storing retrieved data; and (2) the Data visualization and distribution module, namely the smartphone, that retrieves data from the Arduino board and then, it tags, packs and remotely transmits them. ItsPhone is built for the Android platform [5] that has the advantages of offering an open source operating system, highly attractive to users. The interface between Arduino and the Android device is implemented through the Google Accessory Development Kit (ADK) standard [6].

Data collection and pre-processing. Data from the CAN bus are read by an ELM327 ScanTool device through the OBD standard. The ScanTool device is then connected to the Arduino board through a serial connection over which it triggers the retrieval of the following parameters: the engine revolutions per minute, the vehicle speed, the ABS status, the oxygen sensor output voltage, the engine coolant temperature, etc. Kinematics data can serve traffic management purposes in order to monitor road traffic and predict congestion, while other data enable car diagnosis from a remote site, to detect possible malfunctions and collect vehicle statistics.

The Arduino board also collects data from other sensors. Currently, our prototype foresees interaction with $(i)$ homemade low-cost seat-mounted capacitive proximity sensors located in the vehicle to monitor the passengers load, and (ii) humidity, temperature, and pollution sensors located outside the vehicle for environmental monitoring purposes. These sensors periodically transfer measured data to the Arduino board, augmented with Xbee modules to support Zigbee communication.

Including the first type of sensors is motivated by the fact that, while being equipped with built-in front seat occupancy sensors, most vehicles are unequipped with rearseat occupancy sensors. Detecting and remotely transmitting the seat occupancy status can serve several purposes, e.g., facilitating the planning of rescue operations from remote centers, as a matter of fact hazard notifications messages in [7] also carry this information; providing statistics about passengers in public vehicles (taxi, bus, mini-bus) for ticketing and service planning. The second type of sensors could be useful for monitoring the air quality in urban environment and correlating it with traffic congestion patterns. Work is underway to augment the platform with the collection of biometric data from drivers and passengers.

Data visualization and distribution. The smartphone, a Samsung Nexus A in our prototype, is connected to the Arduino board through a USB host interface. Data from the Arduino are complemented with information gathered by the mobile phone itself, e.g., GPS coordinates, so that the platform can geo-reference data also in case of non GPS-equipped cars. Some of the retrieved data are also shown to the end-user

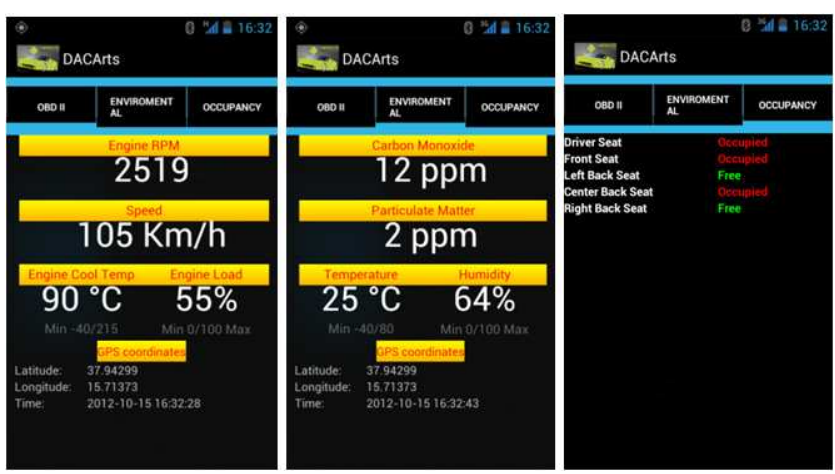

Fig. 2. A snapshot of the developed Android-based ItsPhone application.

through the Android GUI in Fig. 2.

Collected data are separately queued based on their priority and lifetime that are set by the application processing them, and then they are opportunistically remotely transmitted through cellular or Wi-Fi technologies. Data are always sent through Wi-Fi whenever an Access Point is detected. Otherwise, when the cellular connection is only available, the decision whether to transmit data or not is taken according to their lifetime. We identified three data types: high-priority, medium priority, and delay-tolerant data. High-priority data reporting information about a sudden vehicle fault are immediately transmitted over the most reliable and lowest-latency radio interface. Backlogged medium priority packets that are going to expire before a Wi-Fi hotspot is detected (e.g., in a few seconds for traffic signs adaptation, and in some minutes for long-term traffic statistics) are transmitted across the cellular network whenever their time to expiry equals the worst-case transfer time over the cellular technology. Instead, delay-tolerant data concerning the vehicle status, to be sent to car manufacturers and workshops for monitoring and diagnosis purposes, can be stored in the smartphone until the vehicle is in range of a Wi-Fi network or the user owning the device enters her home/office network.

The built prototype confirms the technological feasibility of the conceived platform and preliminary conducted tests prove its effectiveness in offloading the cellular network, thanks to the increasing number of Wi-Fi hotspots deployed across modern cities. Further efforts are required to design business models that incentive the user participation in data collection.

\section{REFERENCES}

[1] J. Zaldivar, et. al., "Providing Accident Detection in Vehicular Networks Through OBD-II Devices and Android-based Smartphones," 5th IEEE Workshop On User MObility and VEhicular Networks 2011.

[2] R. Araujo, et. al., "Driving Coach: A Smartphone Application to Evaluate Driving Efficient Patterns," IEEE Intelligent Vehicles Symposium, 2012.

[3] K. Ali, et. al., "CrowdITS: Crowdsourcing in intelligent transportation systems," IEEE WCNC 2012

[4] http://www.arduino.cc/

[5] http://www.android.com/

[6] http://developer.android.com/tools/adk/index.html

[7] ETSI TS 102 637-3, "Intelligent Transport Systems (ITS); Vehicular Communications; Basic Set of Applications; Part 3: Specifications of Decentralized Environmental Notification Basic Service," 2010. 